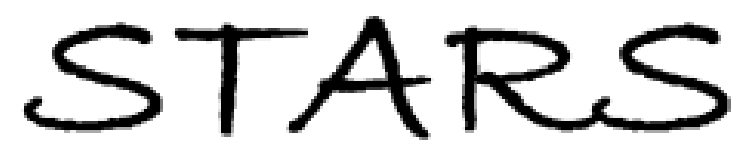

University of Central Florida

STARS

$1-1-2006$

\title{
Density functional theory study of water adsorption at reduced and stoichiometric ceria (111) surfaces
}

Santosh Kumar

University of Central Florida

Patrick K. Schelling

University of Central Florida

Find similar works at: https://stars.library.ucf.edu/facultybib2000

University of Central Florida Libraries http://library.ucf.edu

This Article is brought to you for free and open access by the Faculty Bibliography at STARS. It has been accepted for inclusion in Faculty Bibliography 2000 s by an authorized administrator of STARS. For more information, please contact STARS@ucf.edu.

\section{Recommended Citation}

Kumar, Santosh and Schelling, Patrick K., "Density functional theory study of water adsorption at reduced and stoichiometric ceria (111) surfaces" (2006). Faculty Bibliography 2000 s. 6321.

https://stars.library.ucf.edu/facultybib2000/6321

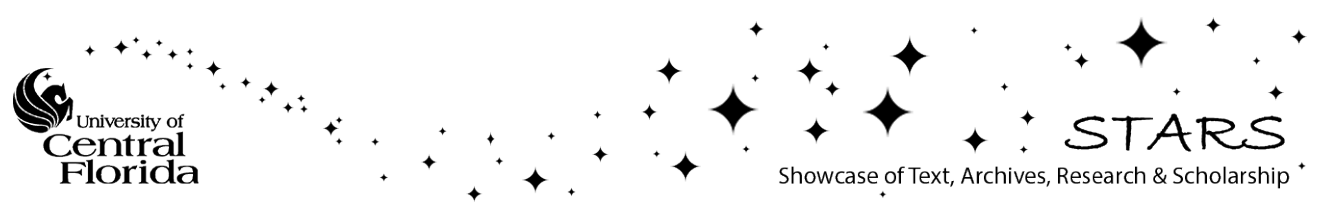




\section{Density functional theory study of water adsorption at reduced and stoichiometric ceria (111) surfaces}

Cite as: J. Chem. Phys. 125, 204704 (2006); https://doi.org/10.1063/1.2400034

Submitted: 28 July 2006 . Accepted: 24 October 2006 . Published Online: 27 November 2006

Santosh Kumar, and Patrick K. Schelling

\section{ARTICLES YOU MAY BE INTERESTED IN}

Hydrogen activation, diffusion, and clustering on $\mathrm{CeO}_{2}$ (111): A DFT+U study

The Journal of Chemical Physics 141, 014703 (2014); https://doi.org/10.1063/1.4885546

A consistent and accurate ab initio parametrization of density functional dispersion correction (DFT-D) for the 94 elements H-Pu

The Journal of Chemical Physics 132, 154104 (2010); https://doi.org/10.1063/1.3382344

Atomic and electronic structure of unreduced and reduced $\mathrm{CeO}_{2}$ surfaces: A first-principles study

The Journal of Chemical Physics 120, 7741 (2004); https://doi.org/10.1063/1.1688316

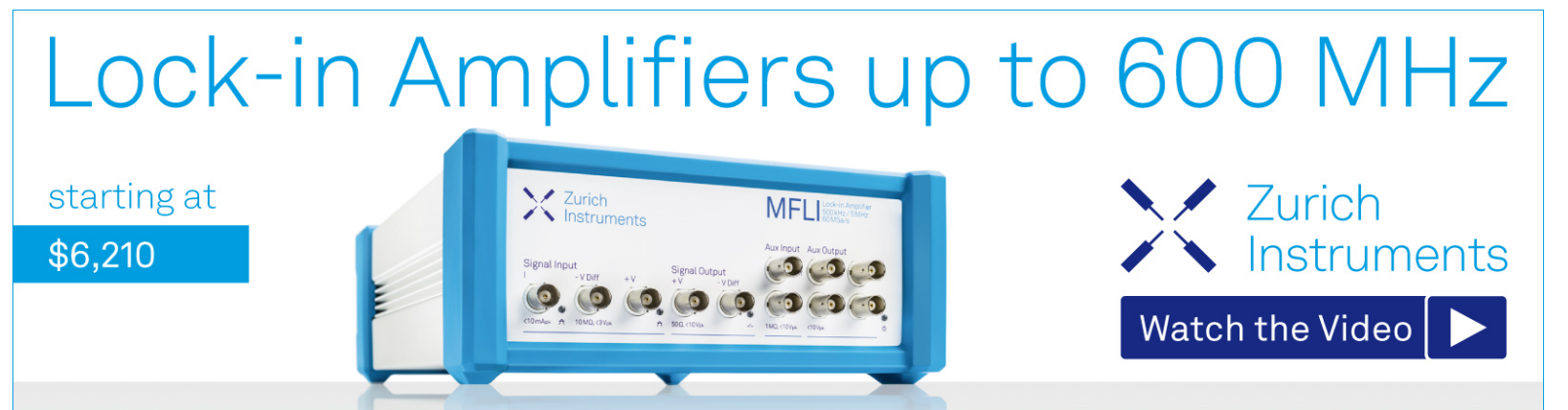

J. Chem. Phys. 125, 204704 (2006); https://doi.org/10.1063/1.2400034 


\title{
Density functional theory study of water adsorption at reduced and stoichiometric ceria (111) surfaces
}

\author{
Santosh Kumar and Patrick K. Schelling ${ }^{\text {a) }}$ \\ Advanced Material Processing and Analysis Center, University of Central Florida, Orlando, \\ Florida 32816 and Department of Physics, University of Central Florida, Orlando, Florida 32816
}

(Received 28 July 2006; accepted 24 October 2006; published online 27 November 2006)

\begin{abstract}
We study the structure and energetics of water molecules adsorbed at ceria (111) surfaces for 0.5 and 1.0 ML coverages using density functional theory. The results of this study provide a theoretical framework for interpreting recent experimental results on the redox properties of water at ceria (111) surfaces. In particular, we have computed the structure and energetics of various absorption geometries at the stoichiometric ceria (111) surface. We find that single hydrogen bonds between the water and the oxide surface are favored in all cases. At stoichiometric surfaces, the water adsorption energy depends rather weakly on coverage. We predict that the observed coverage dependence of the water adsorption energy at stoichiometric surfaces is likely the result of dipole-dipole interactions between adsorbed water molecules. When oxygen vacancies are introduced in various surface layers, water molecules are attracted more strongly to the surface. We find that it is very slightly energetically favorable for adsorbed water to oxidized the reduced (111) surface with the evolution of $\mathrm{H}_{2}$. In the event that water does not oxidize the surface, we predict that the effective attractive water-vacancy interaction will result in a significant enhancement of the vacancy concentration at the surface in agreement with experimental observations. Finally, we present our results in the context of recent experimental and theoretical studies of vacancy clustering at the (111) ceria surface. (0) 2006 American Institute of Physics. [DOI: 10.1063/1.2400034]
\end{abstract}

\section{BACKGROUND AND INTRODUCTION}

The behavior of water at oxide surfaces is critical to many areas of science and technology. For example, in many catalyst applications, water is often present either as a reactant or a spectator. Electrochemical reactions often occur at the interface between water and an oxide passivation layer. The chemistry of water at oxide surfaces is also critical to gas sensors and geochemistry. To begin to understand the complex behavior of these systems, it is essential to develop an atomic-scale description of structure and dynamics.

In recent years, atomic-scale simulations have increasingly been used to fill this important need. For example, first-principles methods based on density functional theory (DFT) have been used to study water at various oxide surfaces, including, for example, dissociative and molecular adsorptions at $\mathrm{TiO}_{2},{ }^{1-3} \mathrm{SnO}_{2}$, and $\alpha$-alumina ${ }^{5}$ surfaces. In addition to DFT studies, there have also been inventive and useful applications of classical molecular dynamics (MD) simulations. ${ }^{6-8}$ More recently, there has been an effort to apply self-consistent tight-binding (SCTB) models to this area, with the important goal of treating systems on a longer length and time scale than traditional DFT approaches. ${ }^{9,10}$ In summary, atomic-scale simulation is an important tool for understanding the properties of water-oxide interfaes.

Ceria is an important material for support of metallic nanoparticles for catalyst applications. ${ }^{11}$ One especially important application of ceria is for catalysis of the water-gas shift (WGS) reaction, described by the overall reaction,

${ }^{a)}$ Electronic mail: pschell@mail.ucf.edu

$$
\mathrm{CO}+\mathrm{H}_{2} \mathrm{O} \rightarrow \mathrm{CO}_{2}+\mathrm{H}_{2} .
$$

Ceria is a very good catalyst support material due to its ability to easily shift oxidation states. ${ }^{12,13}$ Recently, experiments have shown that redox chemistry of water at ceria surfaces may depend strongly on the particular surface orientation. In particular, early work suggests that under most conditions water will tend to oxidize reduced ceria films and powders. ${ }^{14-16}$ In contrast to these studies, there is a report of water causing further reduction of a thin ceria film on a $\mathrm{Pt}$ (111) surface. ${ }^{17}$ However, these studies did not offer the possibility of systematically studying the relationship between chemistry and surface morphology. Recently, more carefully controlled experiments have revealed that, in fact, the (111) face is likely different from other low-index faces. ${ }^{18}$ In particular, water appears to further reduce rather than oxidize reduced (111) ceria surfaces. Experiments have shown that the vacancy concentration at the (111) surface increases in the presence of water vapor. As noted by the authors of Ref. 18 , this presents the interesting possibility that materials with more than one facet exposed to a reducing/oxidizing environment may undergo simultaneous reduction and oxidation reactions. This observation may have significant implications for catalyst applications.

In this paper, we apply DFT calculations to elucidate the behavior of water at the ceria (111) surface. In the case of stoichiometric surfaces, we find that a single hydrogen bond with oxygen on the surface is favorable, resulting in a binding energy in good agreement with experimental temperature-programed desorption (TPD) results. ${ }^{18}$ When an oxygen vacancy is added to the surface, the water molecule 
binds more strongly to the surface indicating an effective attractive interaction between water molecules and oxygen vacancies. We find, in general agreement with experiment, ${ }^{18}$ that the ground-state energy of water in the presence of an oxygen vacancy at the (111) surface is quite low, and watervacancy complexes might be stable with respect to oxidation reaction with the evolution of $\mathrm{H}_{2}$ gas. In particular, we find that oxidation of the surface by water is only weakly exothermic. By calculating the energetics of vacancies at the surface both in the presence and absence of a low concentration of water molecules, we are able to predict the magnitude of the enhancement of vacancy concentration at the surface due to a layer of adsorbed $\mathrm{H}_{2} \mathrm{O}$ molecules.

In the next section, we detail the theoretical method, in particular, DFT, applied to this problem. Section III describes the results obtained for oxygen vacancies at ceria surfaces and the adsorption of water molecules at stoichiometric and reduced ceria (111) surfaces. In Sec. IV we conclude with a discussion of the implications of our results and some possible directions for future inquiry.

\section{THEORETICAL METHODOLOGY}

In recent years it has been shown that DFT methods can be successfully applied to lanthanide and actinide compounds. ${ }^{19}$ The ability to deal with lanthanide and actinide compounds was the result of a careful application of existing and well-established techniques, including ultrasoft pseudopotentials and gradient corrections to the exchange and correlation functionals. For example, it has been shown that DFT can be used to describe several lanthanide compounds including $\mathrm{CeO}_{2} \cdot{ }^{19}$ More recently, the projectoraugmented wave method ${ }^{20}$ (PAW) with gradient-corrected functionals has been applied to lanthanides including $\mathrm{CeO}_{2}{ }^{21,22}$ The combination of the PAW approach with generalized gradient approximation (GGA) for the exchangecorrelation energy represents an accurate and computationally efficient approach for studying lanthanide oxides such as $\mathrm{CeO}_{2}$.

Most of the basic technical details of the calculation described in this article are identical to those used in a previous study of stoichiometric and reduced ceria surfaces. ${ }^{21}$ In particular, the Kohn-Sham DFT calculations were performed using the Vienna $a b$ initio simulation package (VASP) ${ }^{23-25}$ The PAW method ${ }^{20}$ was implemented. The GGA functional due to Perdew et al. $^{26}$ was used to describe the exchange and correlation interactions. The energy cutoff for the planewave basis set was $408 \mathrm{eV}$. For the cerium atoms, the $5 s, 5 p$, $5 d, 4 f$, and $6 s$ electrons were explicitly included as valence electrons. The oxygen sites included $2 s$ and $2 p$ electrons. The calculations were done allowing for the possibility of electron spin polarization, which, while not important for stoichiometric ceria, may be important for reduced ceria. As described in Ref. 21, this approach yields good agreement with experimental measurements for the lattice parameter and bulk modulus of ceria. In particular, we find that the lattice parameter computed by VASP is $5.45 \AA$ compared with the experimental value of $5.41 \AA{ }^{27}$ For the bulk modulus, we obtain a value of $194 \mathrm{GPa}$, compared with the experi-

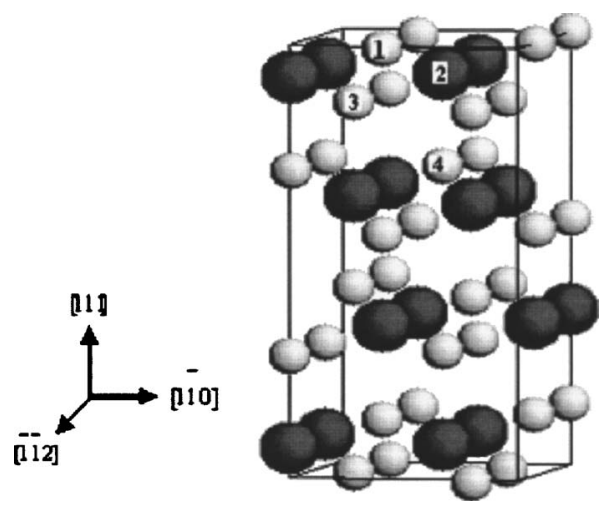

FIG. 1. Slab model used to study the stoichiometric ceria (111) surface. The white and gray spheres represent the $\mathrm{O}$ and $\mathrm{Ce}$ ions, respectively. The actual simulation cell used in our calculations is repeated here along the [112] direction for clarity. The simulation supercell used in the calculations included a total of 24 ions.

mental values of $204 \mathrm{GPa}$ in Ref. 28 and $236 \mathrm{GPa}$ from Ref. 29. The previous ceria calculations ${ }^{21}$ using VASP found a lattice parameter of $5.45 \AA$ and bulk modulus of $193.5 \mathrm{GPa}$, in good agreement with the calculations presented here. Consistent with the observations of Yang et al., ${ }^{21}$ we find that spin polarization has only a small effect on the energetics even for the case of reduced ceria surfaces.

\section{RESULTS}

\section{A. Stoichiometric (111) surface structure and energetics}

Ceria is found in the cubic fluorite structure with eight oxygen neighbors for each cerium ion. The (111) surface of this structure is formed on the most dense lattice plane, and as a result can be expected to be the lowest-energy surface. From a somewhat different point of view, the structure of the (111) surface for the fluorite lattice breaks only one of the eight $\mathrm{Ce}-\mathrm{O}$ bonds characteristic of bulk-coordinated ceria. The structure used in our study to describe the stoichiometric (111) surface is shown in Fig. 1. To simulate a surface, we considered a slab geometry with a vacuum layer of thickness $25 \AA$ added to the simulation cell. For comparison, the work described in Ref. 21 used only $10 \AA$ of vacuum space. However, we found that the energy and structure did not depend significantly on the thickness of the vacuum layer beyond a thickness of $10 \AA$. The structure used in the simulation contained $8 \mathrm{Ce}$ ions and $16 \mathrm{O}$ ions. To perform integration of the electronic states across the Brillouin zone, we used Mokhorst-Pack grids. ${ }^{30}$ We found that a Monkhorst-Pack mesh of $4 \times 4 \times 1 k$ points was adequate for the convergence of the electronic structure. Using this $k$-point mesh resulted in a surface energy of $0.042 \mathrm{eV} / \AA^{2}$. By increasing the mesh of $k$ points to $6 \times 6 \times 1$ the surface energy changed by only $0.05 \%$. Furthermore, the work in Ref. 21 used a $10 \times 10$ $\times 1$ mesh of $k$ points and found $0.037 \mathrm{eV} / \AA^{2}$ for the surface energy, indicating that our much smaller mesh results in quite reasonable accuracy. Another recent DFT study using VASP obtained results very close to the results presented here. In particular, in Ref. 31 the surface energy of the (111) surface was found to be $0.043 \mathrm{eV} / \AA^{2}$. For the stoichiometric 
TABLE I. The displacement of ions along [111] directions due to relaxation of the stoichiometric ceria [111] surface. Atom numbers correspond to those in Fig. 1.

\begin{tabular}{cc}
\hline \hline Ion number & $(111)$ displacement $(\AA)$ \\
\hline 1 & -0.011 \\
2 & 0.040 \\
3 & -0.007 \\
4 & 0.027 \\
\hline \hline
\end{tabular}

(111) surfaces, our results are closer to those of Ref. 31 than those in Ref. 21. However, the differences are quite small and the overall qualitative picture is very consistent. There are no experimental results for the surface energy that can be used for comparison.

In agreement with Ref. 21, we found that the magnitude of the surface relaxations for the stoichiometric (111) surface is very small. We show in Table I the displacements of the ions along the (111) direction for the first few layers. The ion numbers in Table I correspond to the labels in Fig. 1. The very small displacements are consistent with the fact that, as several different studies have shown, the (111) surface is the most stable of the low-index surfaces. Two recent DFT calculations have shown relaxations of similar magnitude to our results. $^{21,31}$ In qualitative agreement with recent DFT results, we find a slight decrease in the separation between the outermost $\mathrm{Ce}$ and $\mathrm{O}$ planes. ${ }^{21,31}$ Studies using empirical potentials tend to result in qualitatively similar behavior. ${ }^{32}$ We find that ionic relaxation lowers the surface energy by less than $10^{-3} \mathrm{eV} / \AA^{2}$, in good agreement with previous studies. ${ }^{21,31}$

\section{B. Atomic structure and energetics of single vacancies on the (111) surface}

By removing an oxygen atom from ceria, a vacancy site will be created. As a result of the oxygen vacancy, electrons are believed to localize on the cerium ions neighboring the vacancy site in a $4 f$ orbital state, thereby reducing the cerium ions from $\mathrm{Ce}_{4+}$ to $\mathrm{Ce}_{3+}$ ions and populating a defect state near the bottom of the conduction band. ${ }^{21}$ Experimental studies have imaged the reduced (111) surface using scanning tunneling microscopy (STM). ${ }^{13,33}$ One of the important conclusions of experimental observations and DFT analysis is that oxygen vacancies tend to group together in clusters. ${ }^{13}$ For the present study, however, we consider only the case of isolated oxygen vacancies. Due to the limited size of the simulation cell (see Fig. 1), in fact, the apparent vacancy concentration is quite high. However, we do not consider vacancy clusters as was done in Ref. 13.

As in Ref. 21, we consider removing a single oxygen atom each of the first three oxygen layers. In particular, referring to Fig. 1, the oxygen ions removed correspond to ion 1 for the outermost layer, 3 for the second layer, and 4 for the third layer. We compute the zero-temperature vacancy formation energy $E_{\mathrm{vac}}$ from the equation

$$
E_{\mathrm{vac}}=E\left(\mathrm{CeO}_{2 \mathrm{vac}}\right)+1 / 2 E\left(\mathrm{O}_{2}\right)-E\left(\mathrm{CeO}_{2}\right) \text {, }
$$

where $E\left(\mathrm{CeO}_{2 \mathrm{vac}}\right)$ is the total energy of the slab with the vacancy, $E\left(\mathrm{CeO}_{2}\right)$ is the total energy of the slab computed
TABLE II. Computed $E_{\mathrm{vac}}$ in $\mathrm{eV}$ for the ceria (111) slab compared with the results in Ref. 21

\begin{tabular}{ccc}
\hline \hline Vacancy position & Ref. 21 & This work \\
\hline First layer & 3.98 & 3.36 \\
Second layer & 3.80 & 3.24 \\
Third layer & 3.96 & 3.36 \\
\hline \hline
\end{tabular}

for the stoichiometric slab, and $E\left(\mathrm{O}_{2}\right)$ is the computed binding energy for an $\mathrm{O}_{2}$ molecule. Because we are primarily interested in how the vacancy formation energy depends on the location of the vacancy and the resulting relaxed structure, it is acceptable to use this form which neglects the oxygen chemical potential. In general, formation energies for vacancies also depend on the $\mathrm{O}_{2}$ chemical potential which can be related to the $\mathrm{O}_{2}$ partial pressure. For our considerations, it is more significant that we have neglected the vibrational contribution to the energy and hence the formation energies computed using Eq. (2) are zero-temperature quantities. The results of this calculation are shown in Table II along with a comparison to prior theoretical results. Overall, the formation energies found in our study are somewhat lower than found by Yang et al. ${ }^{21}$ We believe that this is due to the fact that we did not fix the atoms in the bottom six layers of the slab as was done in Ref. 21. While there are quantitative differences between our results and those in Ref. 21 , the general trend of the dependence of the vacancy formation energy on the atomic plane where the vacancy resides is verified by our study. In particular, we find in agreement with Ref. 21 that the lowest-energy structure corresponds to the case where the oxygen vacancy is placed in the second layer (i.e., the subsurface layer of oxygen).

One of the most striking results that was not noted explicitly in Ref. 21 is the extremely large outward relaxation of $1.07 \AA$ for the oxygen ion just below a vacancy created in the outermost layer of oxygen. When an oxygen vacancy is created in the first or outermost layer of oxygen, an oxygen ion from the layer below relaxes until it actually occupies a position beyond (i.e., towards the vacuum) the outermost layer of cerium ions. Because the oxygen ion relaxes outwards to such a large extent, the resulting structure is in somewhat similar to the structure that results when the oxygen vacancy is created in the second (i.e., subsurface) oxygen layer. However, these structures are, in fact, different as can be seen in Fig. 2.

\section{Water adsorption at stoichiometric ceria (111) surfaces}

Water adsorption on the stoichiometric (111) surface is expected to occur above the cerium ions, with the $\mathrm{Ce}-\mathrm{O}$ bond between the surface and the water molecule having a length comparable with the $\mathrm{Ce}-\mathrm{O}$ bond length in bulk ceria. As noted by Henderson et al., ${ }^{18}$ there are three possible configurations for the water molecule to adsorb on ceria (111) surface. We show the possible configurations schematically in Fig. 3. One possibility is that the water may adsorb with the hydrogen atoms pointing directly out from the surface. This configuration, which has been called the $C_{2 V}$ geometry 

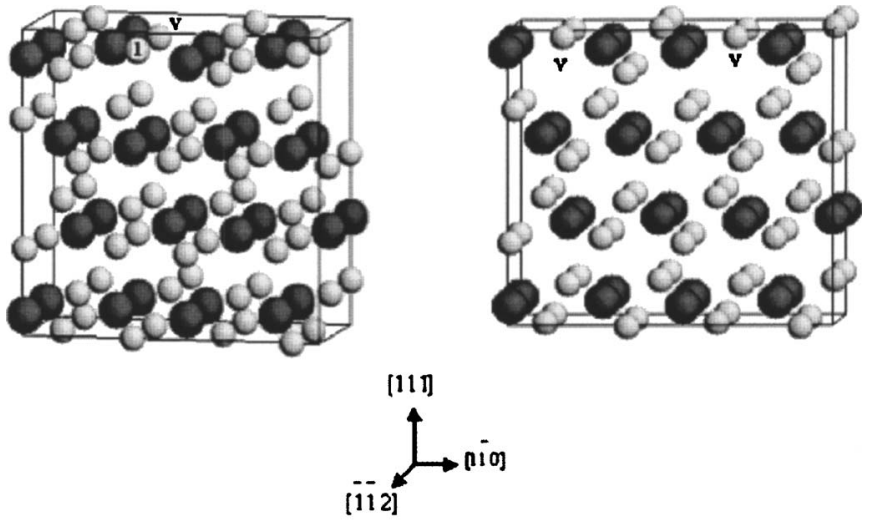

(a)

(b)

FIG. 2. Slab models obtained after the relaxation of reduced ceria (111) surface with oxygen vacancies present in (a) the first layer and (b) the second layer. Oxygen atom present in the first layer and second layer. The "V" represents the position of the oxygen vacancies. In (a), the label 1 indicates the oxygen ion that relaxes outward by $1.07 \AA$.

in reference to the point-group symmetry of the $\mathrm{H}_{2} \mathrm{O}$ molecule, was suggested by Henderson et al. ${ }^{18}$ to be the most likely adsorption geometry. In this case, the symmetry axis of the water molecule is coincident with the [111] surface normal. However, there is no symmetry argument that favors this geometry. In particular, the symmetry of the ceria surface itself corresponds to a $C_{3 V}$ point-group symmetry. As a result, a water adsorbed in the so-called $C_{2 V}$ geometry will experience a different environment for both of the two hydrogen ions. It therefore seems likely that the symmetry axis of the $\mathrm{H}_{2} \mathrm{O}$ molecule will tilt somewhat away from the [111] surface normal. If the tilting is significant, the possibility exists that the water molecule will form one or two hydrogen bonds with surface oxygen ions. These two cases are also shown in Fig. 3.

Using several different initial configurations, we have relaxed the surface with a single water molecule until the largest forces are less than $0.01 \mathrm{eV} / \AA$. The resulting structures and energies show conclusively that a single hydrogen bond is favored over the $C_{2 V}$ geometry and over the case of two hydrogen bonds. We found that starting configurations corresponding to Figs. 3(a) and 3(c) were unstable with respect to forming a single hydrogen bond. For example, beginning with no hydrogen bonds for the $C_{2 V}$ geometry, the water molecule tilts downward to form a single hydrogen bond. As noted previously, this is possible because the surface itself does not share the $C_{2 V}$ symmetry of the water molecule, resulting in a different environment for the two $\mathrm{H}$

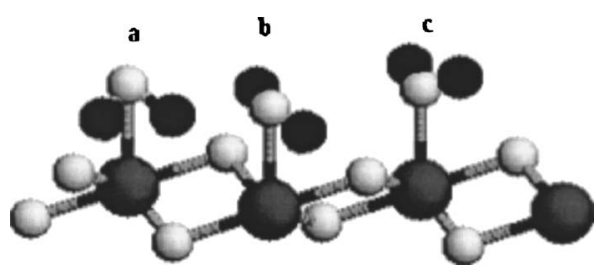

FIG. 3. Schematic representation of the possible configurations for a water molecule adsorbed on a stoichiometric (111) ceria surface. We show in (a) two hydrogen bonds, in (b) a single hydrogen bond, and in (c) no hydrogen bonds corresponding to the so-called $\mathrm{C}_{2 V}$ geometry.
TABLE III. Adsorption energies $E_{\text {ads }}$ and hydrogen bond lengths for $0.5 \mathrm{ML}$ of water adsorbed on the stoichiometric (111) surface for the two different geometries shown in Fig. 4.

\begin{tabular}{lcc}
\hline Geometry & $E_{\text {ads }}(\mathrm{eV})$ & H-bond length $(\AA)$ \\
\hline Fig. 4(a) & 0.56 & 1.94 \\
Fig. 4(b) & 0.58 & 1.74 \\
\hline \hline
\end{tabular}

ions. Similarly, a starting structure with two hydrogen bonds present spontaneously breaks one of the hydrogen bonds to lower the energy of a single hydrogen bond. Because our simulation was a $T=0 \mathrm{~K}$ relaxation, these processes must occur without any activation barrier. As a result, it is not possible to compute a meaningful adsorption energy for the configurations shown in Figs. 3(a) and 3(c). In other words, the only configuration found to be stable in our simulation corresponds to the geometry depicted in Fig. 3(c).

We find that there are two possible final configurations for the adsorbed $\mathrm{H}_{2} \mathrm{O}$ molecule that differ somewhat in energy and structure in spite of the fact that they both possess a single hydrogen bond with the surface. The adsorption energy $E_{\text {ads }}$ of the $\mathrm{H}_{2} \mathrm{O}$ molecule and the bond length for the hydrogen bond for these two structures are given in Table III. The reasons for these differences are made clear by Fig. 4. In fact, the difference arises due to a finite-size effect that results in a difference between the two surface oxygen sites. Because of this finite-size effect, we find that it is slightly more favorable for the hydrogen bond to form with the surface oxygen ion labeled with a number 2 in Fig. 4. In particular, $E_{\text {ads }}$ for the configuration shown in Fig. 4(b) is larger by about $0.02 \mathrm{eV}$ than the configuration shown in Fig. 4(a). There are also small differences in the bond length for the hydrogen bonds between these two configurations. These are also shown in Table III. Because we are primarily interested in the global minima, we will refer in the remainder of the article to the lowest-energy structure [i.e., Fig. 4(b)]. Finally, we find that it is possible to obtain as a final state the structure corresponding either to that shown in Fig. 4(a) or Fig. 4(b) depending on how the initial structure was generated.

The most notable feature seen in experiment is the presence of a fairly strong coverage-dependent desorption energy. ${ }^{18}$ If water indeed forms hydrogen bonds at the surface, competition for bonding sites represents a possible (a)

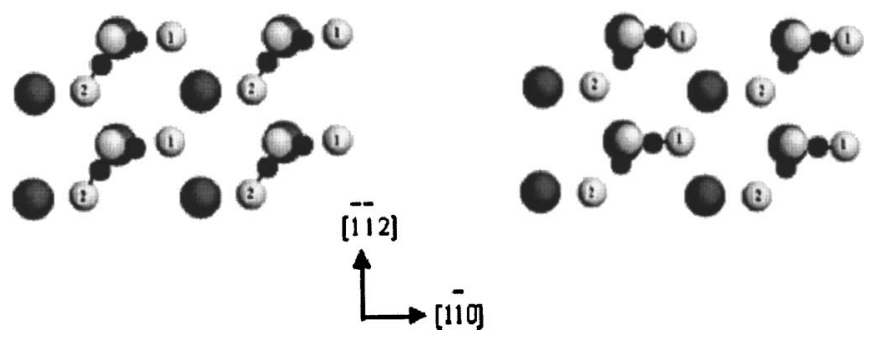

FIG. 4. Two structures studied for $0.5 \mathrm{ML}$ of water adsorbed on the surface. Due to a finite-size effect, these two structures result in slightly different energies. The gray, white, and black spheres represent the $\mathrm{Ce}, \mathrm{O}$, and $\mathrm{H}$ ions, respectively. In addition to the adsorbed water, only the first layers of $\mathrm{O}$ and Ce ions are depicted. 


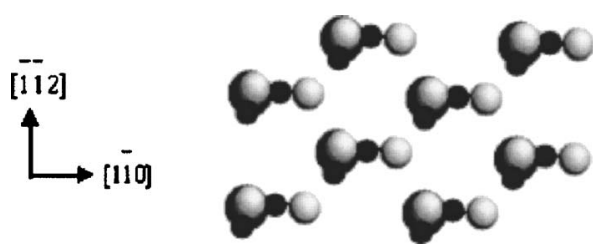

FIG. 5. Computed ground-state structure for 1.0 ML water adsorbed on the (111) surface.

mechanism for the observed coverage dependence. This, however, only seems likely in the event that two hydrogen bonds are formed for each adsorbed water molecule. In particular, each cerium ion at the surface is coordinated with three surface oxygen ions. However, each of the surface oxygen sites is shared by three surface cerium ions. As a result, in the instance that water molecules form double hydrogen bonds with the surface, all available oxygen sites will be saturated at $0.5 \mathrm{ML}$ coverage. Because we predict single hydrogen bonds, it is possible to have 1.0 ML coverage without competition for available oxygen sites. Based on our results, therefore, competition between available sites seems an unlikely explanation of the experimentally observed coverage dependence.

Another possible explanation for the coverage dependence in the experimental TPD results is the increasing importance of water dipole-dipole interactions as the coverage increases. To explore possibility, we studied the energetics of a complete monolayer of water adsorbed to the surface. The second water molecule was added in different starting configurations to a structure with $0.5 \mathrm{ML}$ of water already adsorbed. As with the $0.5 \mathrm{ML}$ case, we find that it is energetically favorable for each adsorbed water molecule to form a single hydrogen bond at the surface. This result was independent of the starting configuration of the second water molecule added to the surface. While the adsorption energy $E_{\text {ads }}$ of the second water molecule is less than that of the adsorption energy of the first water molecule, the differences are very small $(\sim 0.03 \mathrm{eV})$, but possibly large enough to explain the coverage dependence seen in Ref. 18. In particular, the adsorption energy of the second water molecule on the (111) surface was found to be $0.55 \mathrm{eV}$ for the ground-state structure shown in Fig. 5. Finally, we note that an estimate of the dipole-dipole electrostatic interaction energy of neighboring water molecules is about $0.04 \mathrm{eV}$ for each dipole pair. This is comparable to the differences observed in the adsorption energies for the first and second adsorbed water molecules. However, it is important to note that long range dipole-dipole interactions, which may become important at higher concentrations, are not accounted for with the periodic potentials employed in a standard DFT calculation. In other words, there may be long range interactions and correlations that are not appropriately accounted for in our calculations. The computed adsorption energy can be directly related to the experimental value of $0.53 \mathrm{eV}$ determined from an analysis of the TPD experiments. ${ }^{18}$ The good agreement is an indication that the DFT results are providing a realistic description of the system. However, it is important to remember that vibrational effects have been neglected here. A more comprehensive treatment would include either MD simulation or alter-
TABLE IV. Adsorption energy $E_{\text {ads }}$ for $0.5 \mathrm{ML}$ of water on the reduced (111) ceria surface with different positions of $O$ vacancies. Corresponding structures are shown in Fig. 6.

\begin{tabular}{cc}
\hline \hline Vacancy location & $E_{\text {ads }}(\mathrm{eV})$ \\
\hline First layer [Fig. 6(a)] & 0.64 \\
First layer [Fig. 6(b)] & 0.55 \\
Second layer [Fig. 6(c)] & 0.61 \\
Third layer [Fig. 6(d)] & 0.72 \\
\hline \hline
\end{tabular}

nately a calculation of the vibrational modes and an evaluation of the vibrational entropy. However, the fact that the agreement is quite good, along with the fact that the simulation predicts only one adsorption geometry is stable, suggests that the prediction of a single hydrogen bond is an appropriate description for sub-1.0 ML of adsorbed $\mathrm{H}_{2} \mathrm{O}$.

\section{Water adsorption at the reduced ceria (111) surface}

The presence of oxygen vacancies is expected to increase the adsorption energy of water. This is due to the fact that under coordinated $\mathrm{Ce}$ ions will have more electrons available for binding. Another important consideration is that oxygen vacancies are, at least locally, in some sense comparable to positively charged defects, and as such will tend to attract the negatively charge oxygen ion of the $\mathrm{H}_{2} \mathrm{O}$ molecule. In particular, by removing an oxygen atom, the site is no longer occupied by a negative ion that tends to repel the oxygen ion in water. Note, however, that vacancies are created by removing a neutral atom, so that overall neutrality is retained. In short, there should be an effective attractive interaction between water molecules and oxygen vacancies. However, it is not clear whether oxygen vacancies at the surface and water molecules form a stable complex or not. It may be that water will oxidize the surface, thereby eliminating oxygen vacancies with the evolution of hydrogen gas. It is therefore evident that there are two competing mechanisms which, depending on relative energetics, can lead to either enhancement or depletion of surface oxygen vacancies (i.e., reduction or oxidation) due to the presence of adsorbed water molecules.

We have studied several possible configurations of water molecules on ceria surfaces in the proximity of a single oxygen vacancy in either the first, second, or third oxygen layers. We have considered several different initial positions of the water molecule on the defective surface, including directly above the cerium ion nearest to the vacancy, and also directly above the vacancy. In each case, the water molecule prefers to be adsorbed directly above the cerium ion rather than above the vacancy site. In Table IV we show the computed $E_{\text {ads }}$ for the $0.5 \mathrm{ML}$ of water on the reduced ceria (111) surface for different $\mathrm{O}$-vacancy positions. The corresponding relaxed structures for different vacancy configurations are shown in Fig. 6. In each case, we find that the water prefers to form a single hydrogen bond with the surface oxygen ions. For the case of a vacancy in the first oxygen layer, we considered two distinct configurations corresponding to Figs. 6(a) and 6(b). Somewhat surprisingly, we find that the structure corresponding to Fig. 6(b) actually leads to a slight de- 
(a)

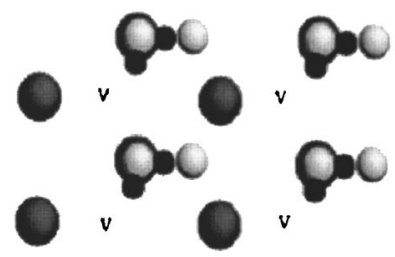

(c)

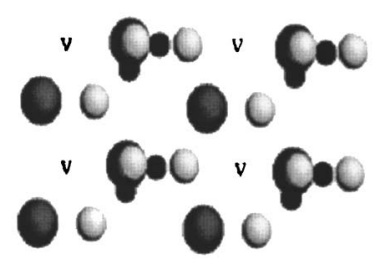

(b)

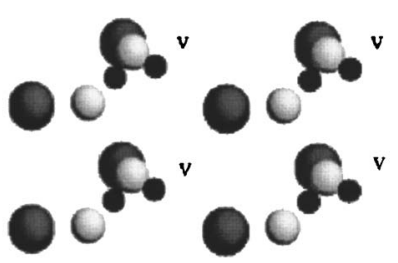

(d)

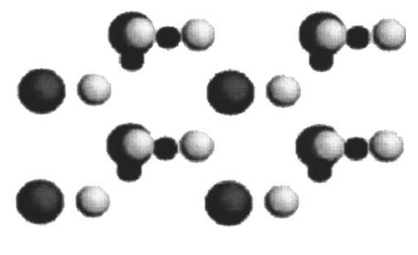

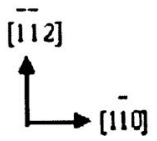

FIG. 6. Structures considered for a single water molecule adsorbed on a surface with a single oxygen vacancy in different locations. The structures (a) and (b) correspond to variants of a single oxygen vacancy in the first oxygen layer. Structure (c) corresponds to a vacancy in the second oxygen layer, and structure (d) corresponds to a vacancy in the third layer directly below the adsorbed water molecule. Only the first layers of $\mathrm{O}$ and $\mathrm{Ce}$ are shown explicitly, although the vacancy sites in each case are represented by a "V."

crease in $E_{\text {ads }}$ when compared to adsorption at the stoichiometric surface. On the other hand, $E_{\text {ads }}$ for the structure in Fig. 6(a) is increased significantly due to the vacancy. We note that for the structure in Fig. 6(a), due to the finite cell size and periodic boundary conditions, there are in effect two vacancies neighboring the water adsorption site. For vacancies located in the second and third oxygen layers, there is a significant increase in $E_{\text {ads }}$. The largest adsorption energy occurs when the oxygen vacancy is in the third layer from the surface and the water molecule is adsorbed on the cerium ion directly above the oxygen vacancy. This structure is shown in Fig. 6(d). In this geometry, the adsorption energy of the $\mathrm{H}_{2} \mathrm{O}$ molecule is $0.72 \mathrm{eV}$. However, while this is the largest adsorption energy that we have found, Fig. 6(d) is not the lowest-energy structure. The ground-state structure for an adsorbed water molecule occurs when the oxygen vacancy is in the second layer from the surface as shown in Fig. 6(c). This is due to the fact that significantly less energy is required to create a vacancy in the second layer compared to the third layer. We compute $E_{\text {ads }}$ of $0.61 \mathrm{eV}$ for the structure in Fig. 6(c). We find that the total energy of the configuration corresponding to Fig. 6(c) is lower by $0.01 \mathrm{eV}$ compared to the structure in Fig. 6(d). This is a very small difference and it seems likely that at finite temperature both configurations will be prevalent. When the oxygen vacancy is in the first layer corresponding to the structure shown in Fig. 6(a), the energy is about $0.08 \mathrm{eV}$ higher than the ground-state energy found for the configuration in Fig. 6(c). The structure shown in Fig. 6(b) is higher in energy by another $0.09 \mathrm{eV}$. This indicates that water adsorption with oxygen vacancies in the first layer of oxygen is substantially less important than ad-

sorption with vacancies in the second and third oxygen layers. However, the energetic differences between the different structures shown in Fig. 6 are comparable to $k_{B} T$ at room temperature, and as a result we expect that each of these structures should be present at some appreciable concentration.

We next consider the enhancement of oxygen vacancies at the surface due to a layer of adsorbed water. This phenomenon has been observed previously in experiment and interpreted to be due to the effective attraction between vacancies and adsorbed water molecules or $\mathrm{OH}$ groups. ${ }^{18}$ The computed increase in the adsorption energy of water on the reduced surface represents an effective attractive interaction between vacancies and adsorbed water molecules. We observe increases in the adsorption energy $\Delta E_{\text {ads }}$ due to vacancies of $0.03 \mathrm{eV}$ for second layer vacancies and $0.14 \mathrm{eV}$ for third layer vacancies. For the present discussion, we assume that vacancies in the first layer are not present in significant concentrations due to the higher energy computed for the structures shown in Figs. 6(a) and 6(b). Assuming that vacancy-vacancy interactions are relatively small, which should be true as long as the effective vacancy concentration is no greater than what we simulate, then the relative increase in oxygen vacancies should be given by a factor $\exp \left(\Delta E_{\text {ads }} / k_{B} T\right)$. For the range of values we have computed for $\Delta E_{\text {ads, }}$, we predict that at room temperature the surface vacancy concentration will increase by a factor of about 3 for second layer vacancies and 300 for third layer vacancies. If the predicted vacancy concentrations become too great, then it is likely that vacancy-vacancy repulsive interactions will limit the accumulation of vacancies, and the predictions by our simple model will be invalid. While the enhancement due to adsorbed water is largest for vacancies in the third layer, the actual concentration of vacancies in the second layer should be somewhat greater. For the first layer, it appears as though there may be very little enhancement except for the case when multiple vacancies are present. In summary, the predicted enhancement in the surface vacancy concentration is a significant effect which may explain the experimental observations. ${ }^{18}$ However, it is also important to note that the experimental TPD spectra do not indicate increased adsorption energies for the water molecules. ${ }^{18}$ In particular, there does not appear to be a significant temperature shift in the desorption peaks due to vacancies. This may point to the possibility that some other stabilization or reduction mechanism is important.

Finally, we have addressed the question of whether water will tend to oxidize the reduced surface. This effect should directly compete with the vacancy stabilization mechanism considered above. To explore this effect, we consider the energy required to oxidize the reduced surface and evolve hydrogen gas $\left(\mathrm{H}_{2}\right.$ molecules). In other words, we compute the energetics of the reaction,

$$
\mathrm{Ce}_{8} \mathrm{O}_{15}+\left(\mathrm{H}_{2} \mathrm{O}\right)_{\mathrm{ads}} \rightarrow \mathrm{Ce}_{8} \mathrm{O}_{16}+\mathrm{H}_{2} \text {. }
$$

The binding energy of the $\mathrm{H}_{2}$ molecule (not including zeropoint energy) was computed to be $4.53 \mathrm{eV}$ in reasonable agreement with experiment and prior DFT calculations. ${ }^{34}$ For the water adsorbed at the reduced surface, if we consider the 
ground-state structure with the oxygen vacancy in the second layer from the surface, the reaction above is predicted to be weakly exothermic. In other words, it is energetically favorable for the water to oxidize the surface with the production of hydrogen gas. The energy evolved during the reaction is predicted to be $0.04 \mathrm{eV}$. However, we stress that these are zero-temperature quantities which may not be representative of the true reaction enthalpy. Nevertheless, the zerotemperature results suggest that while vacancy stabilization by adsorbed water molecules is a significant effect, the resulting clusters are not stable with respect to the oxidation reaction. Another possibility is that water molecules bind even more strongly to vacancy clusters which have not been considered here. This effect might result in water moleculevacancy structures that are stable with respect to oxidation of the surface by water.

Another possibility is that the low-energy structure we found for water adsorbed on the defective surface is not the true ground state. To determine whether or not we have identified the true ground-state structures, we performed MD simulations for water adsorbed at the surface of a reduced ceria surface. Three different starting configurations were studied corresponding to oxygen vacancies in the first, second, and third layers from the surface. The MD simulations were run starting at $T=300 \mathrm{~K}$, and the temperature was gradually reduced to $T=100 \mathrm{~K}$ over a simulation time of 1.15 ps. Finally, the system was relaxed until the forces on the atoms were below $0.05 \mathrm{eV} / \AA$. The final energy and structures obtained were identical to the lowest-energy structures we obtained by relaxation at $T=0 \mathrm{~K}$ and shown in Fig. 6 . When there was a local minimum with a slightly higher energy, the simulated annealing successfully found the lowest-energy structure.

In summary, we predict that adsorbed water will increase the concentration of vacancies at the (111) surface of reduced ceria. This effect is caused by the effective attractive interaction between adsorbed water and oxygen vacancies. The ground-state structure corresponds to a $\mathrm{H}_{2} \mathrm{O}$ molecule adsorbed to the surface with the oxygen vacancy in the second atomic layer from the surface. However, the calculations also predict that oxidation of the surface by water is weakly exothermic. We did not consider vacancy clusters which may bind water even more strongly to the surface. Furthermore, the calculations were performed at zero temperature and hence vibrational and entropic effects were not considered.

\section{DISCUSSION AND CONCLUSIONS}

We have elucidated the structure and energetics of water molecules adsorbed at stoichiometric and reduced ceria surfaces. In all cases, single hydrogen bonds are formed between the water and the surface. We predict that the coverage-dependent TPD spectra observed in experiment are most likely due to dipole-dipole interactions between water molecules. Vacancies increase the adsorption energy of water molecules in most cases. This effect might explain the apparent increase in vacancy concentration observed on the surface in the presence of water vapor. However, we also predict that oxidation of the surface by water to be weakly exothermic. On the other hand, there is a possibility that vacancy clusters could produce an even stronger interaction with adsorbed water molecules. In this case, the water molecules can indeed result in the apparent enhancement of surface oxygen vacancies observed experimentally at the ceria (111) surface. $^{18}$

It is important to consider the limitations of the current simulations. We believe that the reliance on zero-temperature calculations to be the most significant shortcoming. It may be that some qualitative features depend strongly on vibrational and entropic effects. However, another important consideration is the choice of theoretical approach to describe electronic interactions including exchange and correlation effects. For example, the local density approximation (LDA) and GGA approaches may result in a poor description of electronic screening of oxygen vacancies in reduced ceria. In particular, the $f$-electronic orbitals on the cerium ions lie lower in energy than the other conduction band states, and are also usually thought to have very localized orbitals. This has led, for example, Skorodumova et al. ${ }^{22}$ to suggest that a more accurate approach to understanding the reduction of ceria is to place the electrons freed by removing an oxygen for a stoichiometric system into localized $f$ orbitals on two neighboring cerium ions. It has been suggested that the inability of DFT to treat oxidation states from $\mathrm{Ce}_{2} \mathrm{O}_{3}$ to $\mathrm{CeO}_{2}$ within a uniform set of approximations is an indication of the need of a different approach to correctly identify the groundstate electron structure. This led Fabris and co-workers ${ }^{13,35}$ to explore the electronic structure of ceria and reduced ceria using the LDA+U approach, which is believed to be more predictive for strongly correlated electron systems. It is hard to speculate on the significance of strong correlations to the present work. However, it would be important and revealing to repeat a study of water adsorption using the $\mathrm{LDA}+\mathrm{U}$ approach.

\section{ACKNOWLEDGMENT}

This work was supported by an internal University of Central Florida research grant.

${ }^{1}$ P. Lindan, N. Harrison, and M. Gillan, Phys. Rev. Lett. 80, 762 (1998).

${ }^{2}$ A. Kornherr, D. Vogtenhuber, M. Ruckenbauer, R. Podloucky, and G. Zifferer, J. Chem. Phys. 121, 3722 (2004).

${ }^{3}$ P. Lindan and C. Zhang, Phys. Rev. B 72, 075439 (2005).

${ }^{4}$ J. Goniakowski and M. Gillan, Surf. Sci. 350, 145 (1996).

${ }^{5}$ K. Hass, W. Schneider, A. Curioni, and W. Andreoni, Science 282, 265 (1998).

${ }^{6}$ S. Redfern, R. Grimes, and R. Rawlings, J. Mater. Chem. 11, 449 (2001).

${ }^{7}$ S. Kerisit, D. Cooke, D. Spagnoli, and S. Parker, J. Mater. Chem. 15, 1454 (2005).

${ }^{8}$ T. Kundu, R. Rao, and S. Parker, J. Colloid Interface Sci. 295, 364 (2006).

${ }^{9}$ P. Schelling, N. Yu, and J. Halley, Phys. Rev. B 58, 1279 (1998).

${ }^{10}$ J. Halley, Y. Lin, and M. Zhuang, Faraday Discuss. 121, 85 (2002).

${ }^{11}$ Q. Fu, H. Saltsburg, and M. Flytzani-Stephanopoulos, Science 301, 935 (2003).

${ }^{12}$ C. Campbell and C. Peden, Science 309, 713 (2005).

${ }^{13}$ F. Esch, S. Fabris, L. Zhou, T. Montini, C. Africh, P. Fornasiero, G. Comelli, and R. Rosei, Science 309, 752 (2005).

${ }^{14}$ K. Otsuka, M. Hatano, and A. Morikawa, J. Catal. 79, 493 (1983).

${ }^{15}$ C. Padeste, N. Cant, and D. Trimm, Catal. Lett. 18, 305 (1993).

${ }^{16}$ L. Kundakovic, D. Mullins, and S. Overbury, Surf. Sci. 457, 51 (2000).

${ }^{17}$ U. Berner, K. Schierbaum, G. Hones, P. Wincott, S. Haq, and G. Thorn- 
ton, Surf. Sci. 467, 201 (2000).

${ }^{18}$ M. Henderson, C. Perkins, M. Engelhard, S. Thevuthasan, and C. Peden, Surf. Sci. 526, 1 (2003).

${ }^{19}$ C. Pickard, B. Winkler, R. K. Chen, M. C. Payne, M. H. Lee, J. S. Lin, J. A. White, V. Milman, and D. Vanderbilt, Phys. Rev. Lett. 85, 5122 (2000).

${ }^{20}$ P. Bloechl, Phys. Rev. B 50, 17953 (1994).

${ }^{21}$ Z. Yang, T. Woo, M. Baudin, and K. Hermansson, J. Chem. Phys. 120, 7741 (2004).

${ }^{22}$ N. Skorodumova, S. Simak, B. Lundqvist, I. Abrikosov, and B. Johansson, Phys. Rev. Lett. 89, 166601 (2002).

${ }^{23}$ G. Kresse and J. Hafner, Phys. Rev. B 47, 558 (1993).

${ }^{24}$ G. Kresse and J. Furthmuller, Comput. Mater. Sci. 6, 15 (1996).

${ }^{25}$ G. Kresse and D. Joubert, Phys. Rev. B 59, 1758 (1999).

${ }^{26}$ J. Perdew, J. A. Chevary, S. H. Vosko, K. A. Jackson, M. R. Pederson, D.
J. Singh, and C. Fiolhais, Phys. Rev. B 46, 6671 (1992).

${ }^{27}$ H. Whitfield, D. Roman, and A. Palmer, J. Inorg. Nucl. Chem. 28, 2817 (1966).

${ }^{28}$ A. Nakajima, A. Yoshihara, and M. Ishigame, Phys. Rev. B 50, 13297 (1994).

${ }^{29}$ L. Gerward and J. Olsen, Powder Diffr. 8, 127 (1993).

${ }^{30}$ H. Monkhorst and J. Pack, Phys. Rev. B 13, 5188 (1976).

${ }^{31}$ M. Nolan, S. Grigoleit, D. Sayle, S. Parker, and G. Watson, Surf. Sci. 576, 217 (2005).

${ }^{32}$ J. Conesa, Surf. Sci. 339, 337 (1995).

${ }^{33}$ H. Norenberg and G. Briggs, Phys. Rev. Lett. 79, 4222 (1997).

${ }^{34}$ M. Fuchs and X. Gonze, Phys. Rev. B 65, 235109 (2002).

${ }^{35}$ S. Fabris, S. de Gironcoli, S. Baroni, G. Vicario, and G. Balducci, Phys. Rev. B 65, 041102 (2005). 